\title{
Surgical Treatment Outcomes of Spinal Metastases of Nasopharyngeal Carcinoma: The First Report of 30 Patients from a Single Center
}

This article was published in the following Dove Press journal: Cancer Management and Research

\section{Jian Yang* \\ Jinbo $\mathrm{Hu}^{*}$ \\ Da Wang* \\ Qi Jia \\ Jian Jiao \\ Jianru Xiao}

Department of Orthopedic Oncology, Changzheng Hospital, Navy Medical University, Shanghai, People's Republic of China

*These authors contributed equally to this work
Correspondence: Jian Jiao; Jianru Xiao Department of Orthopedic Oncology, Changzheng Hospital, Navy Medical University, Huangpu District, Shanghai,

People's Republic of China

Tel +86 I5221955529

Fax $+8621-81885634$

Email hahameixinmeifei@I63.com; jianruxiao82@I63.com
Background: Studies focused on surgical interventions of spinal metastases of nasopharyngeal carcinoma (SMNPC) are blank.

Methods: Patients with SMNPC who received surgical treatment in our center between 2005 and 2017 were included. Univariate and multivariate analysis of various clinical characteristics and operation-related data were analyzed to identify the independent factors that affected prognosis. Factors with $\mathrm{P}$ values of 0.1 or less were subjected to multivariate Cox regression analysis. $\mathrm{P}$ values of 0.05 or less were considered statistically significant.

Results: A total of 30 patients with SMNPC treated with surgery were included. The thoracic spine was the most frequently involved site. The patients in this series achieved good overall survival (OS, 20.26 months) with limited perioperative complications. The univariate analysis suggested that preoperative Karnofsky performance scale, number of spinal metastases, number of visceral metastases, preoperative Frankel score, surgical resection mode and Ki 67 were potential prognostic factors. In the multivariate analyses, number of visceral metastases, preoperative Frankel score and resection mode were found to be independent prognostic factors.

Conclusion: This is the first study focusing on surgical outcomes in SMNPC. The thoracic spine was the most frequently involved site of SMNPC. Preoperative Frankel score, number of visceral metastasis and surgical resection mode were independent prognostic factors for SMNPC. Combined with adjuvant therapies, surgical interventions should be recommended early when necessary.

Keywords: nasopharyngeal carcinoma, metastasis, spine, prognostic factor, surgical treatment

\section{Introduction}

Nasopharyngeal carcinoma (NPC) is an uncommon tumor throughout the world, and it is the most commonly diagnosed head and neck malignancy in southern China, with an incidence of 25 per $10,000 .^{1-3}$ The widely accepted classification by the WHO divides NPC into 3 categories, I, II and III. ${ }^{4}$ More than $95 \%$ have tumors that fall into WHO type III (undifferentiated), and these are more radiosensitive and have a better prognosis than tumors in other WHO groups. WHO type I and WHO type II tumors are associated with alcohol and tobacco use, are commonly found in nonendemic regions and have a poorer prognosis. ${ }^{4}$ With the application of intensity-modulated radiotherapy (IMRT), the locoregional control of NPC has improved dramatically with an overall survival (OS) of $80-90.5 \%$ at 5 years. ${ }^{5,6}$ Nevertheless, distant metastasis (DM) remains the main reason for therapeutic failure. ${ }^{7-9}$ 
Bone, especially the spine, is the most frequently involved sites in NPC metastases, with an estimated incidence rate of $54-80 \%{ }^{7,10-12}$ As reported, the median OS of patients with spinal metastasis is reported to be 12-23.5 months, ${ }^{13-17}$ much shorter than those without spinal involvement. Obviously, spine involvement indicated a relatively poor prognosis for NPC patients. Though NPC is highly chemosensitive and radiation-sensitive, we found that disease control in some patients with spinal metastases of nasopharyngeal carcinoma (SMNPC) was not satisfactory with the use of chemotherapy, radiotherapy or combination of both. Usually, surgical interventions for SMNPC are rarely applied and mainly

indicated for patients with neurological compression and whose life expectancy is at least 6 months. ${ }^{17}$ However, there is no large series of studies focusing on the surgical interventions for SMNPC because of its rarity.

In this retrospective study, we investigated the surgical treatment outcomes of SMNPC, to identify prognostic factors and to provide possible references for this subgroup.

\section{Materials and Methods Patients and Data Collection}

SMNPC were confirmed by analyzing their disease histories combined with imaging examinations or pathological examination at our institution from January 2005 to December 2017. Informed consent was obtained from all patients or their legal guardians. This research was approved by Ethics Committee of our hospital. Tumor history, general condition (Karnofsky performance scale KPS), metastasis interval time (MIT, interval between the date of NPC diagnosis and the date of diagnosis with SMNPC), number of extraspinal bone metastases, number of vertebral metastases and visceral metastases, pathologic type, preoperative and postoperative neurological assessment based on the Frankel score, ${ }^{18}$ treatment modality, surgery-related and the prognosis data were collected. In addition, serum lactic dehydrogenase (S-LDH), serum alkaline phosphatase (S-ALP) and hemoglobin levels before treatment were collected as well for prognosis analysis.

All patients were followed up on an outpatient basis at a 3-month interval in the first 6 months, and then at a 6-month interval for the remaining time. All patients were followed-up until death or the end of this study.

\section{Criteria for Surgery}

The surgical indications for SMNPC were as follows: (1) pathological fracture, or (2) spinal cord compression, or (3) continuous pain after conservative therapies such as drugs, chemotherapy and radiotherapy, and (4) Tomita score $<8 .{ }^{19}$ Surgical strategies were mainly made according to the Tomita score system, which is recommended widely for spinal metastases. ${ }^{19-21}$

\section{Statistical Analysis}

Survival analysis was performed with SPSS software, version 18.0 (SPSS Inc., Chicago, IL, USA). The postoperative OS was estimated by the Kaplan-Meier method, and univariate analysis for various possible prognostic factors (including age, sex, tumor type, KPS, preoperative Frankel score, spine involvement, number of metastases (multiple vs single), number of metastatic sites (more than one vs one), number of visceral metastases, MIT, treatment modality of the spine metastatic tumors, $\mathrm{Hb}, \mathrm{S}-\mathrm{LDH}$ and S-ALP level and Ki67) was performed by Log rank test. Factors with a $\mathrm{P}$ value $\leq 0.10$ in univariate analysis were subjected to multivariate analysis Cox proportional hazards analysis. P values $\leq 0.05$ were considered statistically significant.

\section{Results}

\section{Patients' Characteristics}

A total of 42 SMNPC patients were recorded between January 2005 and December 2017. Three patients who refused treatment or discontinued treatment halfway and 1 patient lost to follow up were excluded from this study. As the study focused on the surgical outcomes in SMNPC, eight other patients treated with adjuvant therapies were excluded as well. Finally, 30 patients treated with surgery were included and analyzed (Figure 1).

The details of the patients' demographic and clinical characteristics, and treatment approaches are presented in Table 1. The median age was 46.33 years (range: $30-70$ years). Overall, 24 patients (80\%) were male and $6(20 \%)$ were female; $6(20 \%)$ suffered synchronous metastasis, and $24(80 \%)$ had asynchronous metastasis. The median MIT was 25.63 months (range from 0 to 120 months). No or a single visceral metastasis was found in 9 patients (30\%) respectively, while multiple visceral metastases were found in 12 cases (40\%). Single and multiple spine metastasis occurred in 15 patients each $(50 \%)$. The most frequent site of vertebral metastasis was the thoracic spine 


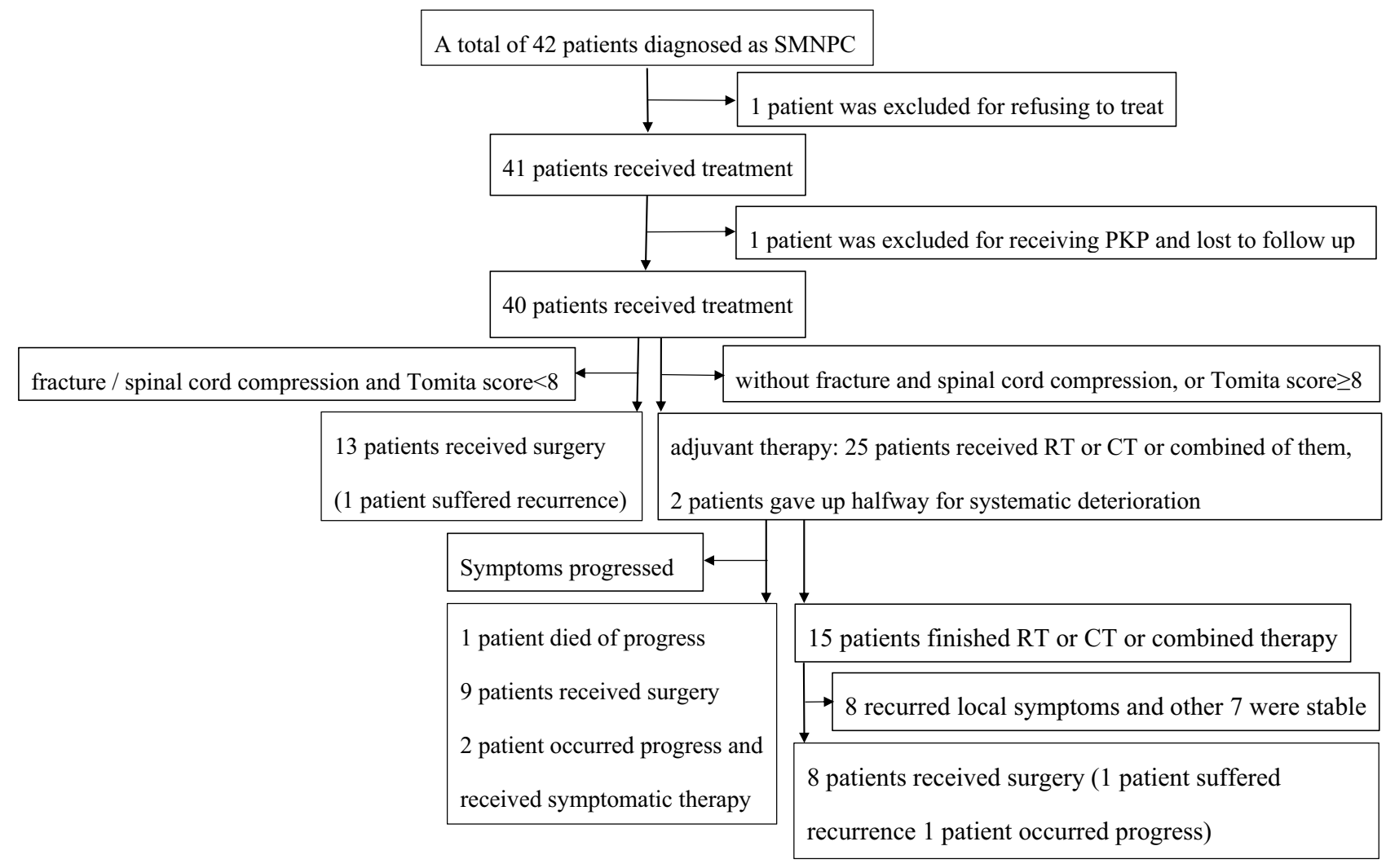

Figure I The patients flow diagram.

(17 cases, 56.67\%), followed by the lumbar spine and cervical spine. Twenty-one (70\%) patients had visceral metastases at admission while $17(56.67 \%)$ were in good general condition according to the Karnofsky Performance Scale (KPS $\geq 80$ ). Histologically, 17 patients were classified as differentiated type, and the remaining 13 were undifferentiated.

Hemoglobin $(\mathrm{Hb})$ less than $11 \mathrm{~g} / \mathrm{dL}$ was found in only 4 cases, and the others had $\mathrm{Hb}$ levels of more than $11 \mathrm{~g} /$ dL. An albumin level (ALB) less than $40 \mathrm{~g} / \mathrm{L}$ was found in 17 cases, while other patients were more than $40 \mathrm{~g} / \mathrm{L}$. The lactate dehydrogenase (LDH) level in $24(80 \%)$ patients was more than $245 \mathrm{IU} / \mathrm{L}$. Alkaline phosphatase (ALP) of the $22(73.33 \%)$ was less than 110 (IU/L). Ki67 of the tumors was less than $30 \%$ in 10 and the others' were greater than $30 \%$ (Table 1 ).

\section{Surgical Treatment of SMNPC}

All 30 patients were treated with surgical interventions, combined with specific adjuvant therapies including chemotherapy, radiotherapy and zoledronic acid. Specifically, 13 patients received surgical interventions directly for pathological fracture and spinal cord compression, and the other 17 patients received surgical interventions after failure of adjuvant therapies. As a result, 9 patients $(30 \%)$ received total en bloc spondylectomy (Figure 2), 11 patients $(36.67 \%)$ received total piecemeal resection, and $10(33.33 \%)$ received debulking or subtotal resection (Table 1). The average blood loss was $1488.89 \mathrm{~mL}$, $1554.55 \mathrm{~mL}$, and $757 \mathrm{~mL}$ in the en bloc, piecemeal and debulking group, respectively, and the average operation time was 327.22 mins, 311.36 mins and 195 mins, respectively. Additionally, all patients received local radiotherapy for primary and metastatic tumors before or after surgical treatment, and most of them (except in 3 cases) received systematic chemotherapy. Moreover, all the patients received bisphosphonates every month for the first year and every two months for the second year to avoid bone-related events.

\section{Survival}

At the last follow-up, two recurrences occurred in the piecemeal group and three cases of local progression occurred in the debulking group after the initial operation. However, secondary spinal cord compression was not detected in these five cases. The use of integrated therapies 
Table I Demographic and Clinical Characteristics of the Series $(n=30)$

\begin{tabular}{|c|c|}
\hline Characteristics & Number of Cases (\%) \\
\hline \multicolumn{2}{|l|}{ Gender } \\
\hline Male & $24(80)$ \\
\hline Female & $6(20)$ \\
\hline Age at admission (yr) & Mean (46) \\
\hline$\leq 46$ & $14(46.67)$ \\
\hline$>46$ & $16(53.33)$ \\
\hline \multicolumn{2}{|l|}{ Pathology type } \\
\hline Differentiated & $17(56.67)$ \\
\hline Undifferentiated & $13(43.33)$ \\
\hline \multicolumn{2}{|l|}{ Preoperative KPS } \\
\hline$<80$ & $13(43.33)$ \\
\hline$\geq 80$ & $17(56.67)$ \\
\hline \multicolumn{2}{|l|}{ Number of visceral metastasis } \\
\hline None & $9(30)$ \\
\hline Single & $9(30)$ \\
\hline Multiple & $12(40)$ \\
\hline \multicolumn{2}{|l|}{ Metastatic level of spine } \\
\hline Cervical & $6(20)$ \\
\hline Thoracic & $17(56.67)$ \\
\hline Lumbar & $6(20)$ \\
\hline Sacrum & I (3.33) \\
\hline \multicolumn{2}{|l|}{ Number of spine metastasis } \\
\hline Single & $15(50)$ \\
\hline Multiple & $15(50)$ \\
\hline \multicolumn{2}{|l|}{ MIT (months) } \\
\hline$<12$ & $14(46.67)$ \\
\hline$\geq 12$ & $16(53.33)$ \\
\hline \multicolumn{2}{|l|}{$\mathrm{Hb}(\mathrm{g} / \mathrm{dL})$} \\
\hline$<11$ & $4(13.33)$ \\
\hline$\geq 1 \mathrm{I}$ & $26(86.67)$ \\
\hline \multicolumn{2}{|l|}{ ALB (g/L) } \\
\hline$<40$ & $17(56.67)$ \\
\hline$\geq 40$ & $13(43.33)$ \\
\hline \multicolumn{2}{|l|}{ LDH (IU/L) } \\
\hline$<245$ & $24(80)$ \\
\hline$\geq 245$ & $6(20)$ \\
\hline \multicolumn{2}{|l|}{ ALP (IU/L) } \\
\hline$<110$ & $22(73.33)$ \\
\hline$\geq 110$ & $8(26.67)$ \\
\hline \multicolumn{2}{|l|}{ Ki67 } \\
\hline$<30 \%$ & $10(33.33)$ \\
\hline$\geq 30 \%$ & $20(66.67)$ \\
\hline
\end{tabular}

(Continued)
Table I (Continued).

\begin{tabular}{|l|l|}
\hline Characteristics & Number of Cases (\%) \\
\hline Treatment & \\
E+A & $9(30)$ \\
P+A & $11(36.67)$ \\
D+A & $10(33.33)$ \\
\hline
\end{tabular}

Abbreviations: KPS, Karnofsky's performance scale; MIT, metastasis interval time; $\mathrm{Hb}$, hemoglobin; ALB, albumin; LDH, lactic dehydrogenase; ALP, alkaline phosphatase; $\mathrm{E}$, en bloc; P, piecemeal; D, debulking; A, adjuvant therapy.

and blockage function of the bone cement in front of the spinal cord might be the main reasons for these findings. Ultimately, 19 deaths $(63.33 \%)$ were recorded. The median OS was 20.26 (range: 4-141) months after operation (Figure 3). The median survival was 34.11, 16.82, 11.6 months in the en bloc, piecemeal and debulking group, respectively. Regarding perioperative complications, one patient suffered from obvious leakage of cerebrospinal fluid and another with a sacral lesion experienced delayed wound healing in the piecemeal group.

Table 2 shows the prognostic values of specific parameters according to the univariate and multivariate Cox regression analyses. The univariate analyses revealed that preoperative KPS ( $p=0.0749$ ), preoperative Frankel score $(\mathrm{p}=0.0037)$, number of visceral metastasis $(\mathrm{p}=0.0182)$, number of spine metastasis $(\mathrm{p}=0.0257)$, Ki67 $(\mathrm{p}=0.09)$ and treatment pattern $(\mathrm{p}=0.0254)$ were statistically significant. In multivariate analysis, adjusting for variables which were significant in univariate analysis, preoperative Frankel score $(\mathrm{p}=0.0094)$, number of visceral metastasis $(p=0.015)$ and treatment pattern $(p=0.029)$ were independent significant factors associated with survival (Figures 4-6).

Other clinical characteristics and laboratory factors $(\mathrm{Hb}$, ALB, LDH, and ALP) were analyzed but were not found to have prognostic value for SMNPC.

\section{Discussion}

\section{Necessity of Surgical Treatment for SMNPC}

Nasopharyngeal cancer (NPC) is predominantly found in southern China. Bone has been thought to be the most common site of metastasis, with an estimated incidence rate of $70-80 \%{ }^{22}$ NPC is highly radiosensitive, and radiotherapy is the mainstay of treatment for NPC, with a $95 \%$ 

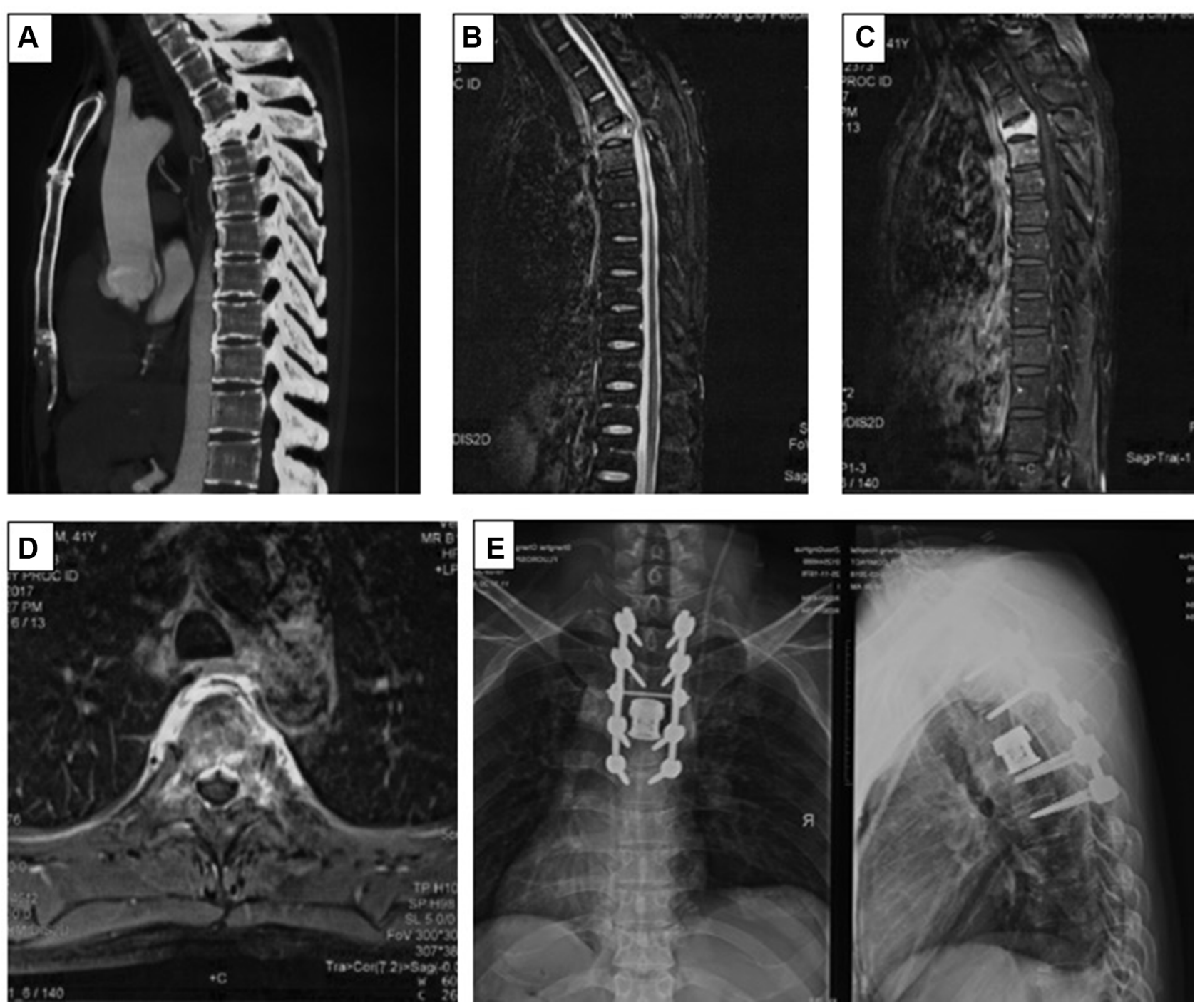

Figure 2 An illustrated SMNPC of total en bloc spondylectomy. (A) CT revealed compressed fracture at T4; (B-D) spinal cord compression and compressed T4 vertebra could be observed on MRI; (E) postoperative X-ray revealed total en bloc spondylectomy of T4 NPC and reconstruction.

Abbreviations: SMNPC, spinal metastases of nasopharyngeal carcinoma; CT, computed tomography; MRI, magnetic resonance imaging.

5-year overall survival. NPC is also highly chemosensitive and platinum-based chemotherapy is the first-line treatment in patients with metastatic NPC. ${ }^{15,16}$ However, we found that chemo-radiotherapy for SMNPC may fail though the primary lesions were controlled well. Shen et $\mathrm{al}^{15}$ found that in NPC patients with bone-only metastases, spine involvement was a strong predictor for unfavorable OS $(\mathrm{p}<0.05)$. Similarly, by studying a large series of bone-only metastasis of NPC, Lu et $\mathrm{al}^{16}$ and $\mathrm{Kumar}^{17}$ also reported that vertebral metastasis was a significant prognostic factor $(p<0.01)$. They also found that the more the spine involvement was, the poorer the prognoses. The reasons may lie in the corresponding complications (movement disorder, paralysis, secondary infection, urination and defecation function disturbance, etc.) of adjuvant therapy failure, followed by shorter survival. Hence, effective treatments for SMNPC are crucial once the failure of radiotherapy and chemotherapy occurs.

\section{Our Experience}

All 30 cases underwent a one-stage operation successfully. Reconstructions were mainly conducted with the combination of titanium cage, artificial vertebra or bone cement and rods-screws system. No failure of internal fixation happened. Among them, 17 patients ( 9 before and 8 after the completion of chemotherapy, radiotherapy or a combination of both, respectively) had to receive surgical interventions due to the disease progression (Figure 1). 


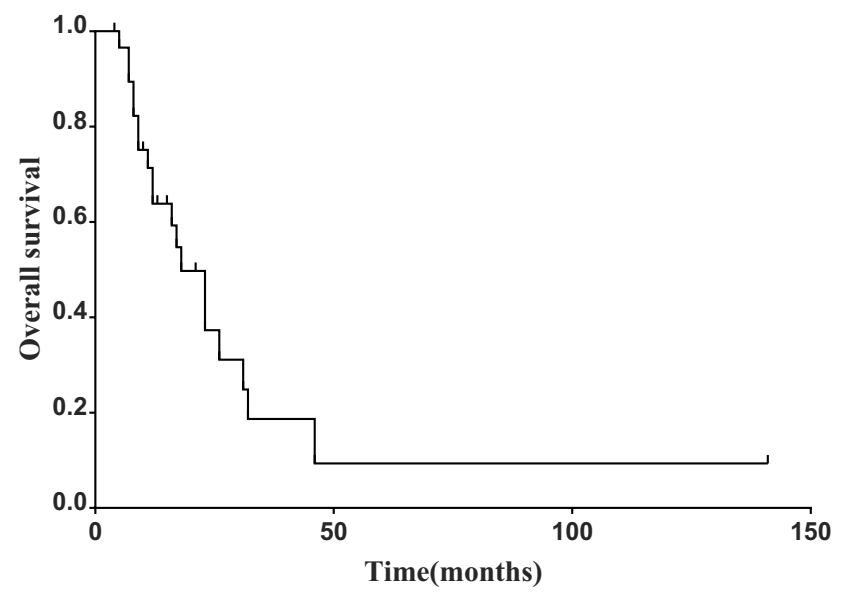

Figure 3 Kaplan-Meier plot showing overall survival of the 30 cases of SMNPC.

Radiotherapy or chemotherapy is not always that effective in the treatment of SMNPC though the high sensitivity to NPC. Surgical interventions were adopted for the goal of saving the neurological function or avoiding neurological deterioration, and prolonging the life expectancy. However, except for some limited mention, ${ }^{17,23}$ no report of large series specifically focusing on the surgical treatment outcomes of SMNPC was found. The present study is the first one that focused on this issue.

As shown in the results, the surgical treatments for SMNPC were safe and the risks were limited and controllable with the updated surgical techniques. For the patients with cerebrospinal fluid leakage, the drainage was removed after controlled drainage without use of lumbar cerebrospinal fluid drainage since the intraoperative suture of dural sac was made. Another patient suffered delayed wound healing, which may have been due to the limited blood supply at the sacrum and the radiotherapy and chemotherapy he received. For the realities and reasons mentioned above, we prefer early surgical interventions for SMNPC patients when conservative therapies seem to be ineffective.

\section{Prognostic Value of Different Elements Value of Epidemiological Elements}

In this study, the patients' median age (46.33 years) was nearly the same as that in previous reports of bone-only metastatic NPC. ${ }^{15,17,24}$ Thus, the series were further divided into two groups ( $\leq 46$ and $>46$ years) for further analysis. The male: female ratio was $4: 1$, indicating a male predominance of SMNPC, which was likely a result of the male predominance in NPC. ${ }^{15,25}$ The prognostic value of age and sex was insignificant $(\mathrm{p}>0.1)$.
As reported, type III NPC has a better prognosis than type I and type II due to its high sensitivity to radiotherapy. ${ }^{17,27}$ Among our SMNPC cases, type III accounted for $43.33 \%$ (13 cases), which is much low than the previously reported $95 \% .{ }^{26}$ According to the statistical analysis, the prognostic value of the pathology type (differentiated vs undifferentiated) was insignificant as well. This pathological difference may have led to the lower radiosensitivity of SMNPC in our study than that has been reported in other studies.

Regarding metastatic onset, Shen et $\mathrm{al}^{15}$ reported that $43.9 \%$ were synchronous and $56.1 \%$ were asynchronous in their study of 312 bone-only metastatic NPCs. In this study, 6 (20\%) metastases were synchronous and the other $24(80 \%)$ were asynchronous, which is quite different from that of Shen's report. The median MIT was 25.63 months, a relatively long interval. Published literature has shown that MIT ( $\leq 24$ vs $>24$ months or $\leq 12$ vs $>12$ months) was a significant prognostic factor $(\mathrm{p}<0.01)$ for bone metastatic NPC. ${ }^{16,25,28,29}$ Nevertheless, this was not confirmed in our SMNPC series.

\section{Value of Serum Parameters}

Elevated LDH is frequently observed in cancer patients. ${ }^{30}$ According to previous literature, NPC patients with abnormally elevated LDH had significantly worse outcome compared with those who had normal LDH level. ${ }^{31,32}$ Similarly, previous researchers found that $\mathrm{Hb}<11.0 \mathrm{~g} / \mathrm{dL}, \mathrm{ALB}<40 \mathrm{~g} / \mathrm{L}$, and $\mathrm{LDH} \geq 245 \mathrm{IU} / \mathrm{L}$ implied poor prognosis in bone-only metastatic NPCs. ${ }^{16,29}$ In another report, ALP $(\leq 110$ vs $>110$ $\mathrm{IU} / \mathrm{L}$ ) appeared to be a significant independent prognostic index in patients with skeletal metastatic NPC. ${ }^{33}$ Nevertheless, no prognostic value was discovered among these above factors in our SMNPC patients.

\section{Potential Prognostic Factors}

Similar to other spine metastatic tumors, ${ }^{24,28}$ the thoracic spine had been reported to be the most frequent site of SMNPC. Furthermore, patients with metastases involving the spine had strikingly poor OS. Notably, those with more than three metastases in the spine had especially poor prognosis. ${ }^{15,17}$ In the current study, univariate analysis also showed that the number of spine metastasis (single vs multiple) was a potential predictor for unfavorable OS among patients with SMNPC ( $\mathrm{p}=0.0376)$, but it was not an independent factor after the multivariate analysis.

The majority of the researchers ${ }^{15,17}$ believed that the KPS was significant for predicting prognosis in cancer 
Table 2 Univariate and Multivariate Analysis of Variables for SMNPC

\begin{tabular}{|c|c|c|c|c|}
\hline \multirow[t]{2}{*}{ Characteristics } & \multicolumn{2}{|l|}{ Univariate Analysis } & \multicolumn{2}{|c|}{ Multivariate Analysis } \\
\hline & HR (95\% Cl) & $P$ value & HR (95\% Cl) & $P$ value \\
\hline $\begin{array}{l}\text { Gender } \\
\text { Male vs Female }\end{array}$ & $1.749(0.603,5.067)$ & 0.303 & & \\
\hline $\begin{array}{l}\text { Age (yrs) } \\
\quad \leq 46 \text { vs }>46\end{array}$ & $1.464(0.590,3.632)$ & 0.411 & & \\
\hline $\begin{array}{l}\text { Pathology type } \\
\text { Di vs UD }\end{array}$ & $1.936(0.745,5.033)$ & 0.175 & & \\
\hline $\begin{array}{l}\text { Preoperative KPS } \\
\quad<80 \text { vs } \geq 80\end{array}$ & $2.23 I(I .889,5.600)$ & 0.0874 & & \\
\hline $\begin{array}{c}\text { Preoperative FS } \\
\text { A,B,C/D,E }\end{array}$ & $4.68 \mathrm{I}(\mathrm{I} .46 \mathrm{I}, \mathrm{I} 4.993)$ & 0.0094 & $4.68(\mid .46 \mathrm{I}-\mathrm{I} 4.99)$ & $0.0094 *$ \\
\hline $\begin{array}{c}\text { Visceral metastasis } \\
N \text { vs } S \text { vs } M\end{array}$ & $2.664(1.277,5.559)$ & 0.009 & $2.58(I .203-5.544)$ & $0.015^{*}$ \\
\hline $\begin{array}{l}\text { Metastatic level } \\
\text { C vs T vs L }\end{array}$ & $0.899(0.496,1.627)$ & 0.725 & & \\
\hline $\begin{array}{l}\text { Count of SMNPC } \\
\text { S vs M }\end{array}$ & $3.185(1.074,9.444)$ & 0.0367 & & \\
\hline $\begin{array}{l}\text { MIT } \\
\qquad<12 \text { vs } \geq 12\end{array}$ & $1.944(0.680,5.558)$ & 0.215 & & \\
\hline $\begin{array}{l}\mathrm{Hb}(\mathrm{g} / \mathrm{dL}) \\
\quad<\mathrm{II} \text { vs } \geq \mathrm{II}\end{array}$ & $2.404(0.528,10.94)$ & 0.257 & & \\
\hline $\begin{array}{l}\text { ALB }(\mathrm{g} / \mathrm{L}) \\
\quad<40 \text { vs } \geq 40\end{array}$ & I.377(0.480, 3.955) & 0.552 & & \\
\hline $\begin{array}{l}\mathrm{LDH}(\mathrm{IU} / \mathrm{L}) \\
\quad<245 \text { vs } \geq 245\end{array}$ & $\mathrm{I} .46 \mathrm{I}(0.46 \mathrm{I}, 4.628)$ & 0.519 & & \\
\hline $\begin{array}{l}\text { ALP }(I U / L) \\
\quad<110 \text { vs } \geq 110\end{array}$ & $1.204(0.425,3.4 \mid 4)$ & 0.727 & & \\
\hline $\begin{array}{l}\text { Ki67 } \\
\qquad 30 \% \text { vs } \geq 30 \%\end{array}$ & $2.688(1.770,9.378)$ & 0.0900 & & \\
\hline $\begin{array}{l}\text { Surgical option } \\
\text { E vs } P \text { vs } D / S\end{array}$ & $2.274(1.209,4.276)$ & 0.0108 & $2.31(1.136-6.421)$ & $0.029 *$ \\
\hline
\end{tabular}

Note: *Indicated statistical significance ( $P$ values $\leq 0.05)$.

Abbreviations: Di, differentiated; UD, undifferentiated; KPS, Karnofsky's performance scale; FS, Frankel score; N, none; S, single; M, multiple; C, cervical; T, thoracic; L, lumbar; SMNPC, spinal metastases of nasopharyngeal carcinoma; MIT, metastasis interval time; Hb, hemoglobin; ALB, albumin; LDH, lactic dehydrogenase; ALP, alkaline phosphatase; E, total en bloc; $\mathrm{P}$, total piecemeal; D/S, debulking/subtotal.

patients. However, this may not be true for all. ${ }^{28}$ In a study including 80 bone-only metastatic NPC patients, Lu et al ${ }^{16}$ found no prognostic value of the KPS. In our study, in the univariate analysis, patients with a preoperative KPS $\geq 80$ tended to have a longer survival than those with a preoperative KPS $<80$ (24 months vs 15 months, $\mathrm{p}=0.0874$ ), while the multivariate analysis results did not reveal significance.
Ki67 is an excellent marker of cell proliferation, ${ }^{34,35}$ and higher Ki67 expression has been found to be associated with poor OS and disease-free survival in NPC. ${ }^{26}$ We divided the SMNPC patients into two groups according to the Ki67 value and found that the group with Ki67 $<30 \%$ had a better prognosis than the group with Ki67 $\geq 30 \%$ (univariate analyses $\mathrm{p}=0.09$ ). However, Ki67 did not indicate significantly predictive power in the multivariate analysis. 


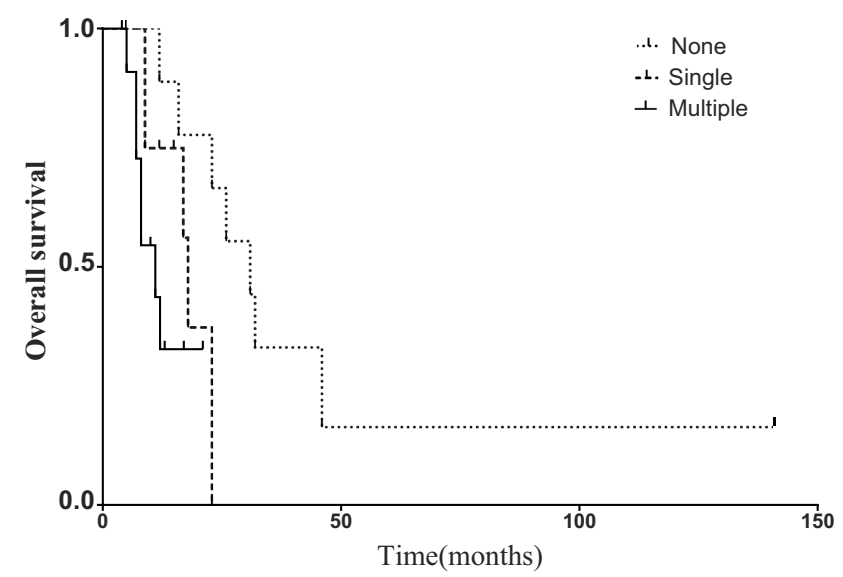

Figure 4 Kaplan-Meier curve for visceral metastasis (None/Single/Multiple).

\section{Independent Prognostic Factors}

Visceral metastasis is important for determining the treatment and prognosis of patients with malignant tumors according to the Tokuhashi score, Tomita score and other literature focused on spine metastases from varied malignant tumors. $^{17,19,36}$ The reason lies in that visceral metastasis indicates a poor systemic status, usually a terminal stage. Additionally, the Frankel score has been broadly accepted as a useful tool to evaluate the neurological function as well as a predictor for late recovery. ${ }^{36,37}$ Remarkably, preoperative Frankel score and the number of visceral metastasis were found to be independent prognostic factors for SMNPC after univariate analysis and multivariate analysis, in accordance with previous studies (Table 2, Figures 4 and 5).

Another independent prognostic factor of SMNPC was the surgical mode. Presently, total en bloc spondylectomy (TES) has been increasingly recognized, and widely

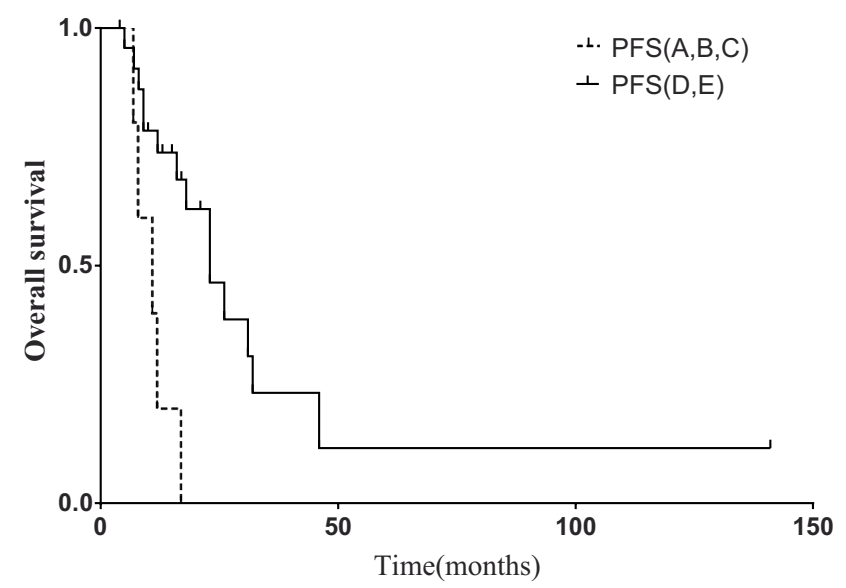

Figure 5 Kaplan-Meier curve for preoperative FS (A, B, C/D, E). Abbreviation: FS, Frankel score.

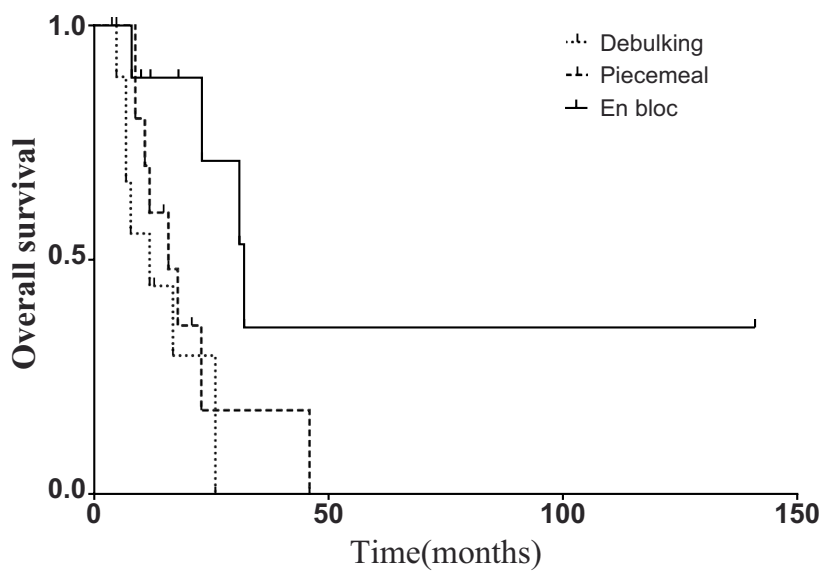

Figure 6 Kaplan-Meier curve for surgical option (En bloc/Total piecemeal/debulking or subtotal).

accepted for the treatment of specific spine tumors and has better tumor control than piecemeal and subtotal resection. $^{38,39}$ In our series, the resection mode included total en bloc spondylectomy, total piecemeal spondylectomy and debulking or subtotal resection, which technique to use was mainly based on the Tomita classification and WBB staging. The final statistical results revealed that TES resulted in better OS than those treated with piecemeal and subtotal mode ( $\mathrm{p}=0.029,95 \%$ CI 1.136-6.421, Figure 6). Since TES is a common and well-developed method for spine tumor therapy, we strongly recommend it when possible for appropriate patients.

\section{Limitations}

Several limitations should be addressed for our series. First is the retrospective nature of the study. Second, our results were concluded from a relatively small sample from a single institution due to the great rarity of SMNPC. Finally, the adjuvant therapies were heterogeneous, which might have a confounding effect. We believe that further studies with large samples and great consistency are needed.

\section{Conclusions}

We report the first study aimed at SMNPC and its surgical treatment, and provide several notable findings. The thoracic spine was the most frequently involved level of SMNPC, while a pathological type predominance was not observed in our series. In addition, preoperative Frankel score, number of visceral metastasis and surgical resection mode may be independent prognostic factors for SMNPC. Combined with chemotherapy and radiation, surgical interventions should be recommended when necessary. 


\section{Disclosure}

The authors declare that they have no conflicts of interest in this work.

\section{References}

1. Wei WI, Sham JS. Nasopharyngeal carcinoma. Lancet. 2005;365 (9476):2041-2054. doi:10.1016/S0140-6736(05)66698-6

2. Jemal A, Bray F, Center MM, et al. Global cancer statistics. $C A$ Cancer J Clin. 2011;61(2):69-90. doi:10.3322/caac.20107

3. De Felice F, Musio D, Magnante AL, et al. Solitary rib metastasis of nasopharyngeal carcinoma. Chin J Cancer Res. 2014;26(2):219-221. doi:10.3978/j.issn.1000-9604.2014.03.02

4. Micheau C, Rilke F, Pilotti S. Proposal for a new histopathological classification of the carcinomas of the nasopharynx. Tumori $J$. 1978;64(5):513-518. doi:10.1177/030089167806400509

5. Lin S, Pan J, Han L, et al. Update report of nasopharyngeal carcinoma treated with reduced-volume intensity-modulated radiation therapy and hypothesis of the optimal margin. Radiother Oncol. 2014;110(3):385-389. doi:10.1016/j.radonc.2014.01.011

6. Peng G, Wang T, Yang K-Y, et al. A prospective, randomized study comparing outcomes and toxicities of intensity-modulated radiotherapy vs. conventional two-dimensional radiotherapy for the treatment of nasopharyngeal carcinoma. Radiother Oncol. 2012;104 (3):286-293. doi:10.1016/j.radonc.2012.08.013

7. Teo PML, Kwan WH, Lee WY, et al. Prognosticators determining survival subsequent to distant metastasis from nasopharyngeal carcinoma. Cancer. 1996;77(12):2423-2431. doi:10.1002/(SICI) 1097-0142(19960615)77:12<2423::AID-CNCR2>3.0.CO;2-N

8. Hui EP, Leung SF, Au JSK, et al. Lung metastasis alone in nasopharyngeal carcinoma: a relatively favorable prognostic group. A study by the Hong Kong Nasopharyngeal Carcinoma Study Group. Cancer. 2004;101(2):300-306. doi:10.1002/cncr.20358

9. Cao C-N, Luo J-W, Gao L, et al. Update report of T4 classification nasopharyngeal carcinoma after intensity-modulated radiotherapy: an analysis of survival and treatment toxicities. Oral Oncol. 2015;51 (2):190-194. doi:10.1016/j.oraloncology.2014.11.009

10. Leung SF, Teo PM, Shiu WW, et al. Clinical features and management of distant metastases of nasopharyngeal carcinoma. J Otolaryngol. 1991;20(1):27-29.

11. Gacani W, Bal IS, Babu MA, et al. Distance metastases from nasopharyngeal carcinoma at Kenyatta Nairobi hospital, Kenya. East Afr Med J. 2001;78(12):678-681. doi:10.4314/eamj.v78i12.8940

12. Bensouda Y, Kaikani W, Ahbeddou N, et al. Treatment for metastatic nasopharyngeal carcinoma. Eur Ann Otorhinolaryngol Head Neck Dis. 2011;128(2):79-85. doi:10.1016/j.anorl.2010.10.003

13. Zhao C-L, Qian G-Q, Chen X-Y, et al. Retrograde analysis of clinical characteristics of bone metastasis in 1031 cases of preliminarily diagnosed nasopharyngeal carcinoma. Asian Pac J Cancer Prev. 2014;15(8):3785-3788. doi:10.7314/apjcp.2014.15.8.3785

14. Jin Y, An X, Cai YC, et al. Zoledronic acid combined with chemotherapy bring survival benefits to patients with bone metastases from nasopharyngeal carcinoma. J Cancer Res Clin Oncol. 2011;137 (10):1545-1551. doi:10.1007/s00432-011-1027-8

15. Shen L, Dong J, Li S, et al. M1 stage subdivision and treatment outcome of patients with bone-only metastasis of nasopharyngeal carcinoma. Oncologist. 2015;20(3):291-298. doi:10.1634/theoncologist.2014-0206

16. Lu T, Guo Q, Cui X, et al. Prognostic evaluation of nasopharyngeal carcinoma with bone-only metastasis after therapy. Yonsei Med J. 2016;57(4):840-845. doi:10.3349/ymj.2016.57.4.840

17. Kumar N, Tan JJH, Zaw AS, et al. Evaluation of scoring systems and prognostic factors in patients with spinal metastases from nasopharyngeal carcinoma. Spine J. 2014;14(12):2946-2953. doi:10.1016/j. spinee.2014.06.001
18. Frankel HL, Hancock DO, Hyslop G, et al. The value of postural reduction in the initial management of closed injuries of the spine with paraplegia and tetraplegia. Paraplegia. 1969;7(3):179-192. doi: $10.1038 /$ sc. 1969.30

19. Tomita K, Kawahara N, Kobayashi T, et al. Surgical strategy for spinal metastases. Spine. 2001;26(3):298-306. doi:10.1097/00007 632-200102010-00016

20. Enneking WF. A system of staging musculoskeletal neoplasms. Clin Orthop Relat Res. 1986;204:9-24.

21. Boriani S, Weinstein JN, Biagini R. Primary bone tumors of the spine. Terminology and surgical staging. Spine. 1997;22 (9):1036-1044. doi:10.1097/00007632-199705010-00020

22. Liu F-Y, Chang JT, Wang H-M, et al. [18 F]fluorodeoxyglucose positron emission tomography is more sensitive than skeletal scintigraphy for detecting bone metastasis in endemic nasopharyngeal carcinoma at initial staging. J Clin Oncol. 2006;24(4):599-604. doi:10.1200/JCO.2005.03.8760

23. Tiruchelvarayan R, Lee KA, Ng I. Surgery for atlanto-axial (C1-2) involvement or instability in nasopharyngeal carcinoma patients. Singapore Med J. 2012;53(6):416-421.

24. Sham JST, Cheung YK, Chan FL, et al. Nasopharyngeal carcinoma: pattern of skeletal metastases. Br J Radiol. 1990;63(747):202-205. doi:10.1259/0007-1285-63-747-202

25. Cao X, Han Y, He L, et al. Risk subset of the survival for nasopharyngeal carcinoma patients with bone metastases: who will benefit from combined treatment? Oral Oncol. 2011;47(8):747-752. doi:10.1016/j.oraloncology.2011.05.010

26. Zhao L, Chen H, Hu B, et al. Prognostic significance of Ki67 expression and the derived neutrophil-lymphocyte ratio in nasopharyngeal carcinoma. Cancer Manag Res. 2018;10:1919-1926. doi:10.2147/CMAR.S167626

27. Mott FE, Ferrarotto R, Nguyen T, et al. Nasopharyngeal carcinoma outcome with induction chemotherapy followed by concurrent chemoradiotherapy. Oral Oncol. 2018;81:75-80. doi:10.1016/j. oraloncology.2018.04.020

28. Rief H, Muley T, Bruckner T, et al. Survival and prognostic factors in non-small cell lung cancer patients with spinal bone metastases: a retrospective analysis of 303 patients. Strahlenther Onkol. 2014;190(1):59-63. doi:10.1007/s00066-013-0431-1

29. Toh C-K, Heng D, Ong Y-K, et al. Validation of a new prognostic index score for disseminated nasopharyngeal carcinoma. Br J Cancer. 2005;92(8):1382-1387. doi:10.1038/sj.bjc.6602525

30. Schwartz MK. Lactic dehydrogenase: an old enzyme reborn as a cancer marker? Am J Clin Pathol. 1991;96(4):441-443. doi:10.1093/ajcp/96.4.441

31. Turen S, Ozyar E, Altundag K, et al. Serum lactate dehydrogenase level is a prognostic factor in patients with locoregionally advanced nasopharyngeal carcinoma treated with chemoradiotherapy. Cancer Invest. 2007;25(5):315-321. doi:10.1080/07357900701209103

32. Jin $Y$, Ye X, Shao L, et al. Serum lactic dehydrogenase strongly predicts survival in metastatic nasopharyngeal carcinoma treated with palliative chemotherapy. Eur J Cancer. 2013;49(7):1619-1626. doi:10.1016/j.ejca.2012.11.032

33. Jin $Y$, Yuan MQ, Chen JQ, et al. Serum alkaline phosphatase predicts survival outcomes in patients with skeletal metastatic nasopharyngeal carcinoma. Clinics. 2015;70(4):264-272. doi:10.6061/clinics/2015(04)08

34. Ahn HK, Jung M, Ha S-Y, et al. Clinical significance of Ki-67 and p53 expression in curatively resected non-small cell lung cancer. Tumour Biol. 2014;35(6):5735-5740. doi:10.1007/s13277-014-1760-0

35. Luporsi E, Andre F, Spyratos F, et al. Ki-67: level of evidence and methodological considerations for its role in the clinical management of breast cancer: analytical and critical review. Breast Cancer Res Treat. 2012;132(3):895-915. doi:10.1007/s10549-011-1837-z

36. Yang M, Xu W, Liu T, et al. Development and validation of a novel survival prediction model in patients with spinal metastasis from non-small cell lung cancer. Spine. 2019;44(4):246-257. doi:10.1097/ BRS.0000000000002816 
37. Zhao C, Zhang Z, Zhong N, et al. Outcomes and prognostic factors for surgically treated patients with breast cancer spine metastases. J Bone Oncol. 2018;12:38-43. doi:10.1016/j.jbo.2018.03.003

38. Huang W, Wei H, Cai W, et al. Total en bloc spondylectomy for solitary metastatic tumors of the fourth lumbar spine in a posterior-only approach. World Neurosurg. 2018;120:e8-e16. doi:10. 1016/j.wneu.2018.06.251
39. Kawahara N, Tomita K, Murakami H, et al. Total en bloc spondylectomy for spinal tumors: surgical techniques and related basic background. Orthop Clin North Am. 2009;40(1):47-63. doi:10. 1016/j.ocl.2008.09.004

\section{Publish your work in this journal}

Cancer Management and Research is an international, peer-reviewed open access journal focusing on cancer research and the optimal use of preventative and integrated treatment interventions to achieve improved outcomes, enhanced survival and quality of life for the cancer patient.
The manuscript management system is completely online and includes a very quick and fair peer-review system, which is all easy to use. Visit http://www.dovepress.com/testimonials.php to read real quotes from published authors. 